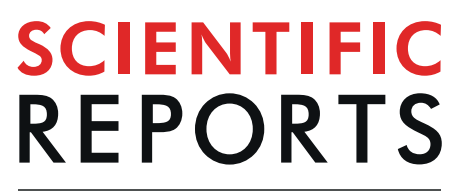

natureresearch

\title{
TH17 cells expressing CD146 are significantly increased in patients with Systemic sclerosis
}

Amira Gabsi ${ }^{1,2^{*}}$, Xavier Heim ${ }^{2,5}$, Akram Dlala $^{1}$, Asma Gati ${ }^{1}$, Haifa Sakhri ${ }^{1}$, Ahmed Abidi ${ }^{1}$, Sonia Amri ${ }^{1}$, Bilel Neili' ${ }^{1}$, Aurelie S. Leroyer ${ }^{2}$, Alexandrine Bertaud ${ }^{2}$, Monia Smiti Khanfir ${ }^{3,4}$, Fatma Said ${ }^{3,4}$, Mohamed Habib Houman ${ }^{3,4}$, Brigitte Granel ${ }^{6}$, Marcel Blot-Chabaud ${ }^{2}$, Nathalie Bardin ${ }^{2,5,7}$ \& Raja Marrakchi ${ }^{1,7}$

Systemic sclerosis (SSc) is an autoimmune disorder characterized by vascular damage, excessive fibrosis and abnormalT cells immune-regulation. CD146 is an adhesion molecule essentially expressed in the vascular system, but also on TH17 lymphocytes. In view of the recently described role of CD146 in SSc, we hypothesized an involvement of CD146 positive TH17 cells in this disease. Compared to healthy controls, we showed that both soluble form of CD146 (SCD146), and IL17A levels were increased in patients with SSc with a positive correlation between both factors. A significant increase in $\mathrm{TH} 17$ cells attested by an increase of ROR T, IL17A mRNA and CD4+ IL17A+ cell was observed in patients with SSc. Interestingly, the percentage of TH17 cells expressing CD146 was higher in patients with SSC and inversely correlated with pulmonary fibrosis. In vitro experiments showed an augmentation of the percentage of TH17 cells expressing CD146 after cell treatment with SCD146, suggesting that, in patients the increase of this sub-population could be the consequence of the SCD146 increase in serum. In conclusion, TH17 cells expressing CD146 could represent a new component of the adaptive immune response, opening the way for the generation of new tools for the management of SSc.

Systemic sclerosis (SSc) is an autoimmune disease characterized by excessive fibrosis in the skin and internal organs, vascular damage and immune dysfunction. Two major clinical subsets, namely limited cutaneous (lcSSc) and diffuse cutaneous (dcSSc) forms have been described according to the extent of skin fibrosis ${ }^{1}$. Pulmonary fibrosis and pulmonary arterial hypertension $(\mathrm{PAH})$, are the most serious complications and constitute currently the major causes of death ${ }^{2,3}$. The study of new molecular targets is essential in the understanding of the physiopathology of the disease, and the establishment of new therapeutic strategies.

Among them, CD146 has been recently proposed as a new molecular target in $\mathrm{SSc}^{4}$. This cell adhesion molecule, also called MUC18 and MCAM, is a membrane glycoprotein belonging to the Immunoglobulin super family ${ }^{5}$. It is ubiquitously distributed on endothelium ${ }^{6,7}$, with a preferential localization at the endothelial junction ${ }^{7}$. CD146 is involved in several functions such as cell adhesion ${ }^{8}$, inflammation ${ }^{9}$, and angiogenesis through different signaling pathways ${ }^{8}$. CD146 also exists as a soluble form (sCD146) in the blood, resulting from the proteolysis of the membrane form ${ }^{9-11}$. In a mice model of SSc induced by bleomycin, we showed that mice lacking CD146 were more susceptible to develop skin fibrosis than wild type animal. Fibrosis development could be prevented by subcutaneous sCD146 injection. We also evidenced the involvement of Wnt pathway since CD146 deficiency was associated with an up-regulation of the canonical Wnt pathway, leading to the profibrotic state . $^{4}$

The expression of CD146 is not restricted to the endothelium since it is also expressed on other cell types such as melanoma cells ${ }^{12}$, extra villous trophoblastic cells ${ }^{13}$ and TH17 cells ${ }^{14}$. TH17 cells are involved in the pathogenesis of several autoimmune diseases including SSc ${ }^{15}$. These cells play a critical role in the pathogenesis of autoimmune diseases, leading to production of several cytokines as IL17A, IL22, IL21 and IL26 ${ }^{14}$. The retinoic acid receptor-related-orphan- receptor-gamma $\mathrm{T}(\mathrm{ROR} \gamma \mathrm{T})$, is the key transcription factor required for TH17 cell

${ }^{1}$ Université de Tunis El Manar, Faculté des Sciences de Tunis, LR05SE05, 2092, Tunis, Tunisia. ${ }^{2}$ Aix Marseille Univ, INSERM, INRA, C2VN, Marseille, France. ${ }^{3}$ Internal medicine service, University hospital center LA RABTA, 1007, Tunis, Tunisia. ${ }^{4}$ Université de Tunis El Manar, Faculté de Medicine de Tunis, 1007, Tunis, Tunisia. ${ }^{5}$ Service d'Immunologie, Pôle de Biologie, Hôpital de la Conception, Assistance Publique-Hôpitaux de Marseille (AP-HM), Marseille, France. ${ }^{6}$ Department of Internal Medicine and Therapeutics, Timone Hospital, Marseille, France. ${ }^{7}$ These authors contributed equally: Nathalie Bardin and Raja Marrakchi. *email: amira.gabsi@gmail.com 


\begin{tabular}{|l|l|}
\hline SSc patients $\mathbf{n}=\mathbf{5 0}$ & Median \\
\hline Median age (years) & $60(28-68)$ \\
\hline Male/female & $7 / 43$ \\
\hline Clinical Features & $\mathrm{n}(\%)$ \\
\hline Limited cutaneous form & $24(48)$ \\
\hline Diffuse cutaneous form* & $26(52)$ \\
\hline Ulcers, scars, gangrene & $17(65)$ \\
\hline Pulmonary fibrosis (PF) & $19(73)$ \\
\hline Pulmonary arterial Hypertension (PAH) & $20(76)$ \\
\hline Digestive involvement & $13(50)$ \\
\hline Kidney involvement & $8(30)$ \\
\hline Immunological Results & $\mathrm{n}(\%)$ \\
\hline Positive for anti-nuclear antibodies & $48(96)$ \\
\hline - centromeric staining (ACA) & $24(47)$ \\
\hline - positive for anti-topoisomerase I (anti-topo I) & $26(49)$ \\
\hline
\end{tabular}

Table 1. [A] Demographic data and disease characteristics of SSc patients. n: number of subjects. *According to the classification of ACR 2013.

differentiation and for production of cytokines which participates in protective immunity at boundary tissues ${ }^{15}$. IL17A primarily binds to IL-17RA ${ }^{16}$ and initiates a pro-inflammatory signal, which is strongly involved in the progression of many autoimmune diseases in humans ${ }^{17,18}$.

In SSc, the mRNA and protein expression of IL17A was found to be positively correlated to an outcome index, the modified Rodnan skin score ${ }^{17,19}$.

In view of the role played by CD146 in SSc, we hypothesized a preponderant role of CD146 positive TH17 cells in SSc. To investigate this hypothesis, we explored in the present study, the concentrations of sCD146 and IL17A in serum of patients with SSc as compared to healthy controls. In parallel, we evaluated the proportion of TH17 cells expressing CD146 (CD146+ TH17) over total TH17 cells and analyzed the potential correlations with clinical events. At last, effect of sCD146 was tested on the proportion of CD146+ TH17 among total TH17cells of patients with SSc.

\section{Materials and Methods}

Ethics statement. All patients gave informed consent and all methods were performed in accordance with the relevant Guidelines and regulations. The Medical Bioethics Committee at the Pasteur Institute of Tunis approved This study under the reference 2015/04/E/FST.

Blood samples. Peripheral blood samples were obtained from 50 patients with SSc admitted to internal medicine departments of university hospital La RABTA Tunis Tunisia; all patients fulfilled the ACR/EULAR Classification Criteria and were then sub classified according to Leroy et al. criteria ${ }^{1}$. The cohort is explained in Table 1. Fifty sera of age and sex matched blood controls (median age 61 (57-70) and sex ratio 10 male/40 female).

Separation of blood cells on a FICOLL density gradient. Peripheral blood mononuclear cells (PBMCs) were prepared by centrifugation over a Ficoll-Hypaque Gradient (Histopaque; Sigma Diagnostics, St Louis, MO) and cryopreseved in 10\% dimethyl sulphoxide (DMSO) in fetal bovine serum (FBS). Sera were also collected and stocked in $-20^{\circ}$ until use.

Soluble CD146 detection. We measured serum sCD146 concentration using a capture enzyme-linked immunosorbent assay kit (CYQUANT ELISA, Biocytex Marseille, France) according to the manufacturer's protocol. The ELISA assay was validated previously $y^{4}$. A plastic support coated with specific mouse monoclonal anti human $\mathrm{CD} 146 \mathrm{~F}(\mathrm{ab})_{2}$ fragments binds to the sCD146 to be measured.

Next the mouse monoclonal antibody anti-CD146 coupled with peroxidase binds to a remaining free antigenic determinant of the CD146. The bound enzyme peroxidase is then revealed by its activity in a predetermined time on the TMB substrate. After stopping the reaction, the intensity of the signal is directly related to the concentration of sCD146 initially contained in the sample.

Quantification of IL17A. IL17A was quantified in serum of healthy controls and patients with SSc, using commercially available ELISA kit (Quantikinie ELISA human IL-17A immunoassay R\&D Systems France). All the amount of cytokine present in diluted serum will be captured. After several washes to remove the excess, a polyclonal antibody bound to an enzyme specific for human IL17A is added. After washes, the reaction is revealed with a substrate (TMB), giving a color whose intensity is proportional to the amount of IL17A bound and the plates are read at $450 \mathrm{~nm}$ and optical density were converted to concentration using a four parametric logistic regression.

mRNA quantification. RNA isolation and cDNA synthesis. Total cellular RNA was extracted from cryopreseved PBMCs using the acid guanidine-phenol- chloroform technique with Trizol (GIBCO, BRL, Bethesda, $\mathrm{MD})$, according to the manufacturer's instruction. After incubation for $10 \mathrm{~min}$ at $65^{\circ} \mathrm{C}$, RNA samples were stored 


\begin{tabular}{|c|c|c|}
\hline Primer & & $\operatorname{Tm}^{\circ}$ \\
\hline$\beta$-actin & $\begin{array}{l}\text { 5' CATCCTGTCGGCAATGCCAGG 3' (forward primer) } \\
5^{\prime} \text { CTTCTTGGGC ATGGAGTCCTG 3' (reverse primer) }\end{array}$ & 54 \\
\hline ROR $\delta \mathrm{T}$ & $\begin{array}{l}\text { 5'CTTCCTCAGCGCCCTGTGGTT3' (forward primer) } \\
\text { 5' CCCAGGACGGTTGGCATTGA 3' (reverse primer) }\end{array}$ & 68 \\
\hline IL17A & $\begin{array}{l}5^{\prime} \text { GCAAGAGATCCTGGTCCTGA } 3^{\prime} \text { (forward primer) } \\
5^{\prime} \text { AGCATCTTCTCGACCCTGAA } 3^{\prime} \text { (reverse primer) }\end{array}$ & 63.8 \\
\hline CD146 & $\begin{array}{l}5^{\prime} \text { CCCGGGCCACCATGGGGCTTCCCA 3' (forward primer) } \\
5^{\prime} \text { GGATCCTCACCGGCTCTCCGGCTC } 3^{\prime} \text { (reverse primer) }\end{array}$ & 72.4 \\
\hline
\end{tabular}

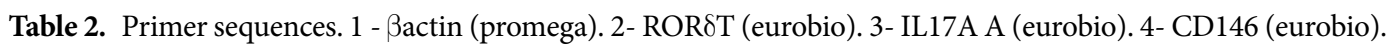

at $-80^{\circ} \mathrm{C}$. Total RNA quality and quantity was assessed by absorbance at $260 \mathrm{~nm}$ using a nucleic acid and protein analyzer. This RNA was reverse-transcribed in a total volume of $25 \mu \mathrm{L}$ with 200 units of Moloney murine leukemia virus reverse transcriptase (Invitrogen, US) in the presence of $1.5 \mu \mathrm{L}$ of $5 \mathrm{X}$ First Strand Buffer (Invitrogen, US) (250-mM Tris-HCL; 375-mM KCL; 15-mM MgCL2) in addition to 10 units of RNAsine (Invitrogen, US), $1.25 \mu \mathrm{L}$ of $100 \mathrm{mM}$ DTT and $1.25 \mu \mathrm{L}$ of each desoxynucleotide triphosphates (Promega, US) at 10-Mm. After that, all these reagents were incubated at $37^{\circ} \mathrm{C}$ for $60 \mathrm{~min}$ and then cooled at $-20^{\circ} \mathrm{C}$. The integrity and the quantity of the cDNA samples were tested by amplifying $\beta$-actin cDNA.

Quantitative RT-PCR. Real-time PCR was performed on a The Step-One-Plus Real-Time PCR (Applied Biosystems, CA, USA) using the SYBR Green qPCR Kit (Power Up ${ }^{\mathrm{TM}}$ SYBR $^{\mathrm{TM}}$ Green Master Mix A25742 Applied Biosystem Thermofischer FRANCE), and continuous fluorescence monitoring. A reaction volume of $25 \mu \mathrm{l}(1.0 \mu \mathrm{l}$ cDNA) was amplified for 40 cycles after initial denaturation $\left(95^{\circ} \mathrm{C}, 10 \mathrm{~min}\right)$ with the following parameters: $95^{\circ} \mathrm{C}$ for $10 \mathrm{~s}, 60^{\circ} \mathrm{C}$ for $15 \mathrm{~s}$, and $72^{\circ} \mathrm{C}$ for $20 \mathrm{~s}$. Samples were run in duplicate, and relative quantification of mRNA level was performed using $\beta$-actin as an endogenous reference. We obtained the primer sequences (Table 2 ) from literature and checked through PCR in silico. We used three primers: ROR $\gamma$ T, IL17A and CD146. qPCR data was analyzed by calculating the fold difference individually for each gene. Cycle threshold (Ct) is defined as the number of PCR cycles at which the fluorescence signal rises above the threshold value and is inversely proportional to the amount of template present in the reaction. Ct values of genes and control samples were compared and the fold difference calculated like: Fold $\mathrm{CHANGE}=2-\Delta \mathrm{Ct}$ where $\Delta \mathrm{Ct}=\mathrm{CT}$ sample- $\mathrm{CT}$ endogenous control. The $\mathrm{Ct}$ values were plotted, assuming the same threshold for all genes evaluated. Ct values $<40$ were only used for calculation of the PCR efficiency. (This was explained in Step-one-plus book).

Flow cytometry analysis. PBMCs were prepared by centrifugation over a Ficoll technique and cryopserved in $10 \%$ SVF- DMSO. Thawed cells were routinely tested for viability by trypan blue exclusion. For cytokine secretion assays, PBMCs were stimulated with complete cell culture medium (Eurobio France) with 1/1500-inonomycine. - 1/2000 PMA - 1/3000 Golgi Stop overnight. Then cells were washed twice with 5\% fetal bovine serum (FBS)-phosphate-buffered saline (PBS) prior to staining. First of all, cells were incubated for ten minutes with $\mathrm{F}_{\mathrm{c}}$ blocking (Human TrueStain $\mathrm{FcX}^{\mathrm{TM}}$ Ozyme FRANCE) as an isotype control. Then, PBMCs were stained with up to five antibodies for $30 \mathrm{~min}$ at room temperature. After staining, the cells were fixed with $2 \%$ formaldehyde-PBS prior to analysis on a BD canto flow cytometry (BD Bioscience, San Diego, CA, USA). Lymphocytes were gated based on forward-scatter and side-scatter properties; at least 10,000-gated events were analyzed using the Flow Software (Flow LLC Ashland, Oregon). We calculated absolute cell percentages based on flow cytometry data and complete blood counts. Six colors were used to analyze the surface phenotype and intracellular cytokine production of PBMCs. The antibodies used were CD3 PerCP Cy5/CD4 APC-Cy7/CD25 PE-Cy7/CD146 Alexa fluor 488 for cell surface staining and IL17A PE and FOXP3 APC for intracellular staining and cytokine staining.

Flow cytometry analysis was performed on the canto 2 flow cytometry (BD Bioscience, San Diego, CA, USA). Lymphocytes subsets were gated by first forward versus side light scatter to identify lymphocytes, then CD4+ T cells were gated using a dot plot showing CD3 versus CD4. First of all, TH17 cells were identified using a dot plot showing CD4 and IL17A and then TH17 cells expressing CD146 were gated using a dot plot showing IL17A and CD146. Frequencies of cells were determined using statistical option in FLOWJO V10.5.3.

In vitro sCD146 stimulation on PBMCs from patients with SSc. Fresh PBMCs from patients were used. $5 \times 10^{5}$ cells from each patient were incubated with $100 \mathrm{ng} / \mathrm{ml}$ of sCD146 [RPMI-1640 (Gibco, Carlsbad, CA, USA) with $5 \% \mathrm{AB}+$ human serum, $10 \mathrm{mM}$ HEPES, non-essential amino acids, sodium private, $2 \mathrm{mM}$ L-glutamine (Sigma, St. Louis, MO, USA), 100 units $/ \mathrm{ml}$ of penicillin and $100 \mu \mathrm{g} / \mathrm{ml}$ of streptomycin (Invitrogen, Carlsbad, CA, USA)] $+100 \mathrm{ng} / \mathrm{ml} \mathrm{sCD} 146$, then cultured in a humidified incubator at $37^{\circ} \mathrm{C}$ with $5 \% \mathrm{CO} 2$ for 24 hours. For cytokine secretion assays, cells were stimulated overnight with PMA-Ionomycine-Golgi Stop culture medium. And the cells were stained with CD3 PerCP Cy5/CD4 APC-Cy7/CD146 Alexa fluor 488 antibodies for cell surface staining and IL17A PE antibody for cytokine staining.

Flow cytometry analysis was performed on the canto 2 flow cytometry (BD Bioscience, San Diego, CA, USA). Lymphocytes subsets were gated as described above in flow cytometry analysis section.

Finally, using these cells stimulated or not with sCD146, total RNA was extracted and cDNA was synthesized as described above (2.6) and Q-RT PCR was performed using CD146 primers (Table 2). 
A)

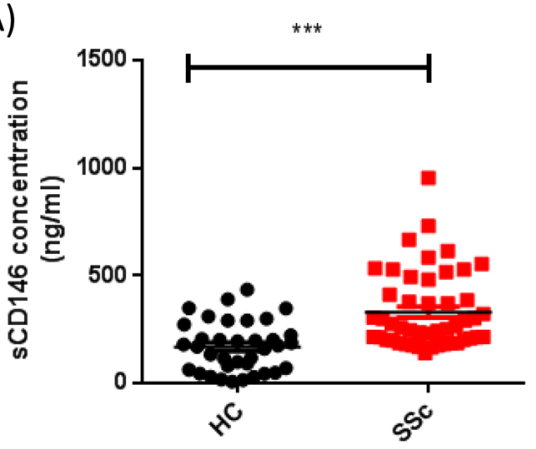

C)

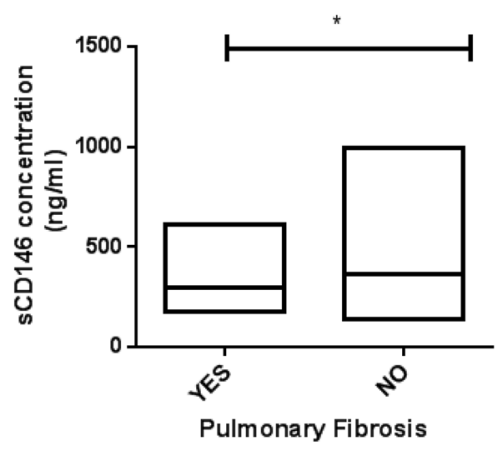

B)

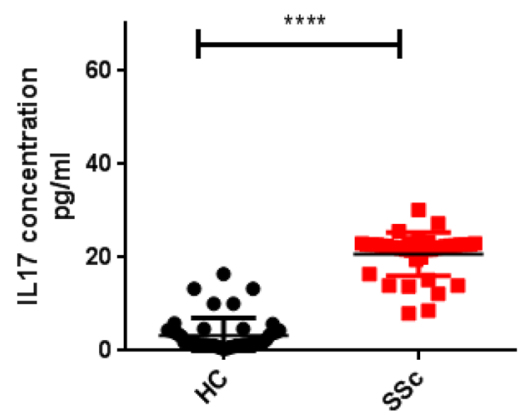

D)

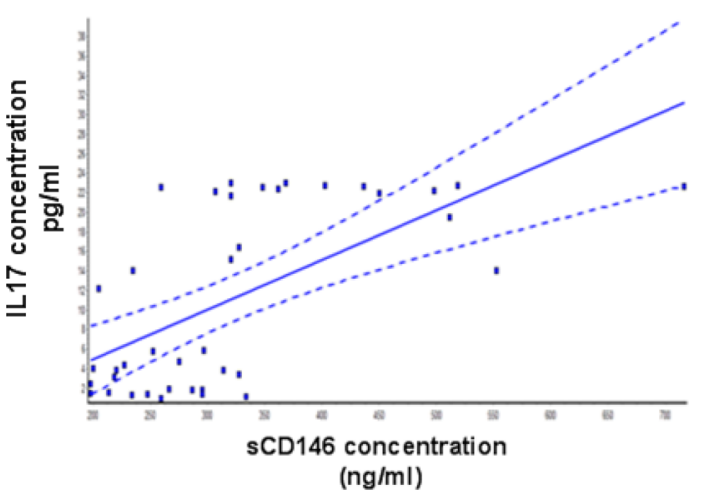

Figure 1. Serum level of soluble CD146 (sCD146) and circulating IL17A in patients with Systemic sclerosis (SSc) and healthy controls (HC). (A) Soluble CD146 (sCD146) concentration was significantly higher in patients with SSc $(\mathrm{n}=50)$ than in $\mathrm{HC}(\mathrm{n}=50)$ [329.0 $\pm 24.90 \mathrm{ng} / \mathrm{ml}$ versus $168.4 \pm 19.39 \mathrm{ng} / \mathrm{ml}, \mathrm{p}<0,0001]$. (B) The cytokine IL17A concentration was significantly increased in patients with SSc $(\mathrm{n}=50)$ than in (HC) $(\mathrm{n}=50)[20.05 \pm 1.97 \mathrm{ng} / \mathrm{ml}$ versus $3.32 \pm 0.54 \mathrm{ng} / \mathrm{ml}, \mathrm{p}<0,0001]$. (C) A significant association was observed between pulmonary fibrosis $(n=19)$ and low levels of $\mathrm{sCD} 146(\mathrm{p}=0.0147)$. (Yes = have pulmonary fibrosis/ No = have other involvement than pulmonary fibrosis). (D) A correlation was observed between the IL17A and soluble CD146 (sCD146) concentrations in patients with SSc $(\mathrm{p}<0.0001)$.

Statistical analysis. Results presented as means \pm standard deviation. Statistical analyses were performed using Prism (Graph Pad Software, San Diego, CA). A p value $<0.05$ was considered statistically significant.

Correlation and linear regression are the most commonly used techniques for quantifying the association between two parameters. The analyses are based on linear relationship between the two variables. A scatter plot is essential before embarking on any correlation-regression analysis to show that this is indeed the case. In our study, correlation and linear regression let us study correlation between sCD146 concentrations, IL17A concentration and TH17 expressing CD146 cells percentages.

\section{Results}

Epidemiologic study. We included 50 patients with SSc from the internal medicine department of university hospital RABTA Tunis, having a score of 9 or higher according to the ACR/EULAR criteria. Our cohort was composed of 43 women and 7 men and a mean age of the onset of illness equal to $60 \pm 16$ years [28-68 years]. Rodnan's score ranged from one to 41 in our cohort and averaged 17. Then, clinical data highlighted several inaugural events such as Raynaud's syndrome, skin involvement, arthralgia, cutaneous sclerosis, gastro-esophageal reflux. Pulmonary Arterial Hypertension (PAH), is one of the most serious disorders of systemic sclerosis, and is diagnosed by cardiac Doppler ultrasound after exercise dyspnea. Another serious manifestation is pulmonary fibrosis demonstrated by Chest CT examination. Our cohort was constituted by 20 patients with PAH and 19 patients with pulmonary fibrosis (Table 1 ).

Soluble CD146 and IL17A concentrations are increased in sera of patients with SSc. Serum concentrations of sCD146 and IL17A were measured in patients with SSc $(n=50)$ and compared to age - and sexmatched controls $(n=50)$. As shown in Fig. 1A,B, sCD146 and IL17A concentrations were significantly higher in patients with SSc as compared to healthy controls (respectively, $329.0 \pm 24.90 \mathrm{ng} / \mathrm{ml}$ versus $168.4 \pm 19.39 \mathrm{ng} /$ $\mathrm{ml}, \mathrm{p}<0,0001$ and $20.05 \pm 1.97 \mathrm{ng} / \mathrm{ml}$ versus $3.32 \pm 0.54 \mathrm{ng} / \mathrm{ml}, \mathrm{p}<0,0001)$. Regarding clinical association, we found a significant association between low $\mathrm{sCD} 146$ concentrations and pulmonary fibrosis $(\mathrm{p}=0.04)$ as shown in Fig. 1C, while there was no correlation with other clinical disorders described in Table 1. For IL17A, no correlation was observed with clinical disorders. Interestingly, we found a positive correlation between sCD146 and IL17A concentrations ( $\mathrm{p}=<0.0001)$, as illustrated in Fig. 1D. 

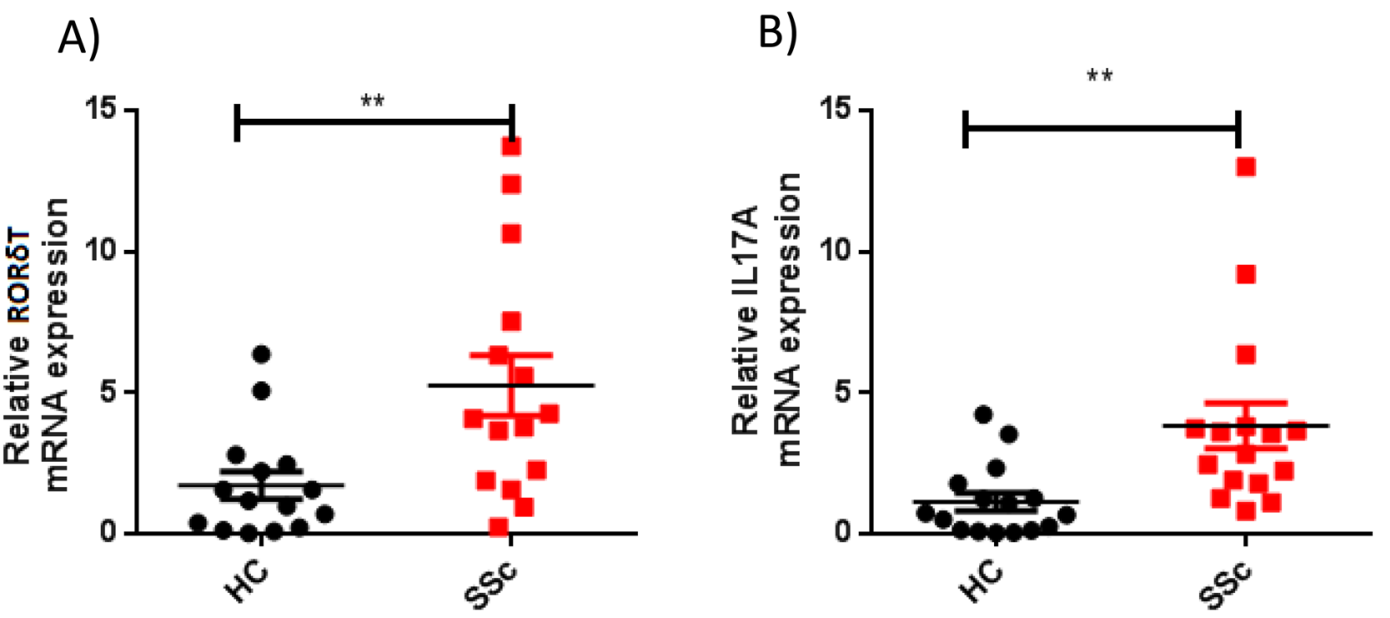

Figure 2. Relative mRNA expression of IL17A and ROR $\gamma \mathrm{T}$ in PBMCs from patients with Systemic sclerosis as compared to healthy control. (A) The expression of the transcription factor ROR $\gamma \mathrm{T}$ mRNA was higher in patients with SSc than in HC $(\mathrm{p}=0,0013)$. (B) The expression of the cytokine IL17A mRNA was higher in patients with SSc than in HC $(\mathrm{p}<0.0001)$.

The number of CD146+ TH17 cells is increased in patients with SSc. We analyzed the expression of IL17A and of the master transcription factor of TH17 cells, ROR $\gamma$ T, in patients with SSc as compared to control group.

The mRNA expression of ROR $\gamma \mathrm{T}$ and IL17A was first quantified by qRT-PCR in peripheral blood mononuclear cells (PBMCs) of patients with SSc and healthy controls. ROR $\gamma$ T and IL17A mRNA expression were significantly higher in patients with SSc as compared to healthy controls ( $\mathrm{p}=0,0013$ and $\mathrm{p}=<0.0001)$ (Fig. 2A,B). For ROR $\gamma \mathrm{T}$ and IL17A mRNA expression, no significant association was observed with clinical features.

In a second step, we studied the proportion of TH17 cells over the total CD4+ cells population using a flow cytometry analysis (Fig. 3A). We found a higher percentage of TH17 cells in patients with SSc (Mean $16.95 \pm 0.8482$ ), as compared to healthy controls (Mean $9.135 \pm 0.2522, \mathrm{p}<0.05$ ) as shown in Fig. 3B.

Then, we analyzed CD146 expression on TH17 cells, we found a higher percentage of TH17 cells expressing CD146 (CD146+ IL17A + subset) in patients with SSc as compared to healthy controls (Fig. 4A,B). Interestingly the percentage of TH17 cells expressing CD146 was inversely correlated to the pulmonary fibrosis (4 C) but no association with other clinical features was detected.

To better understand the link between TH17 cells and sCD146, the ratio between CD146+ TH17 cells and total TH17 cells was calculated (percentage of TH17 cells expressing CD146/TH17 cells). We found a significant correlation between $\mathrm{sCD} 146$ concentration and this ratio $(\mathrm{p}=0,0135)$, as shown in Fig. 4D.

sCD146 affects T lymphocytes in PBMCs from patients with SSc. We evaluated sCD146 effects on TH17 cells after sCD146 treatment (100 ng/ml for 24 hours) of PBMCs of patients with SSc. Interestingly, an up regulation of the percentage of TH17 cells expressing CD146 (CD146+ IL17A+ subset) was found after sCD146 treatment (Fig. 5A). The number of cells was determined at T0, and after $24 \mathrm{~h}$ of sCD146 treatment with 492 million $+/-6088$ at T0 and 491 million $+/-6441$ at T24, no significant difference was observed $(\mathrm{p}=0.8992)$ attesting for the absence of cell proliferation. In addition expression of CD146 was up regulated after SCD146 treatment as demonstrated by fluorescence intensity analysis (Fig. 5B). Besides, after treatment with sCD146, mRNA expression of CD146 was increased in PBMCs treated with sCD146 as compared to untreated cells (Fig. 5C).

\section{Discussion}

In systemic sclerosis, we recently reported a major role of CD146, a molecule ubiquitously distributed on vascular cells, but also expressed on a subset of lymphocytes involved in the pathogenesis of the disease, TH17 cells. In this study we demonstrated for the time that TH17 lymphocytes expressing CD146 are increased in patients with SSc, suggesting their involvement in the pathology.

In these patients, we found a higher percentage of TH17 cells expressing CD146 and a correlation between sCD146 and IL17A serum concentrations. Interestingly the ratio of TH17 cells expressing CD146/Total TH17 cells in patients with SSc was inversely correlated to pulmonary fibrosis. Finally, in vitro treatment of TH17 cells with sCD146 led to an up regulation of CD146 expression. Afterward, PBMCs treatment with sCD146 induced an increase in the mRNA expression of CD146 as compared to untreated cells.

In a previous study, unfavorable evolution of SSc was associated with a decrease of SCD146 concentrations. We recently showed that SCD146 concentrations were increased in patients with SSc and that severe manifestations of the disease, as pulmonary fibrosis, were associated with low sCD146 concentrations $s^{4}$. Patients producing high and sustained levels of SCD146 may be protected against the disease complications, accounting for the reduction in the concentration of the soluble form in the case of pulmonary fibrosis ${ }^{4}$. In the present study, we confirm these data, highlighting sCD146 as a novel biomarker for the assessment of the disease. In addition, we evidence a 
HC

A)

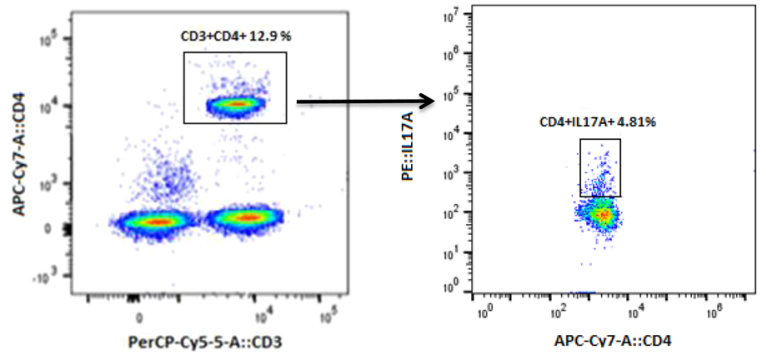

SSc

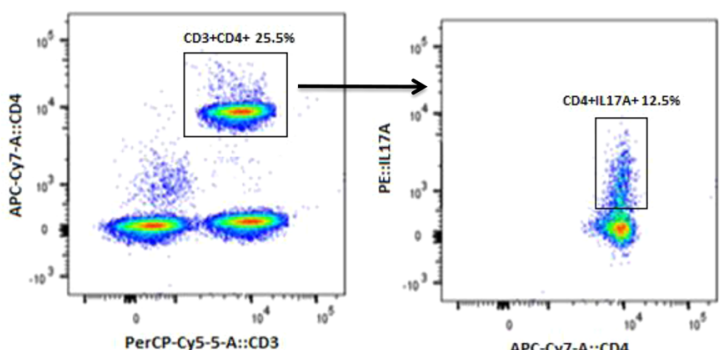

B)

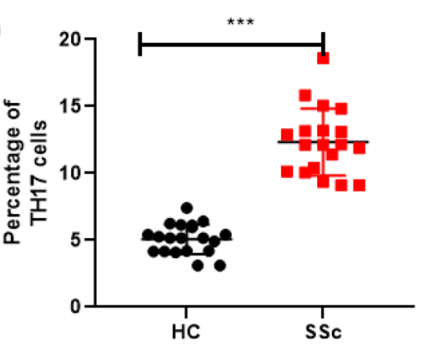

Figure 3. Flow cytometry analysis of TH17 cells in patients with systemic sclerosis as compared to healthy controls. (A) Gating strategy of TH17 cells in HC as compared to PBMC of Patients with SSc by FACS: cells were activated with PMA-Ionomycine-Golgi Stop overnight and $\mathrm{F}_{\mathrm{c}}$ blocking (Humain TrueStain FcX ${ }^{\mathrm{TM}}$ Ozyme FRANCE) was used as an isotype control. (B) The percentage of TH17 cells in patients with SSc was found to be increased as compared to HC $(\mathrm{p}=0.0019)$.
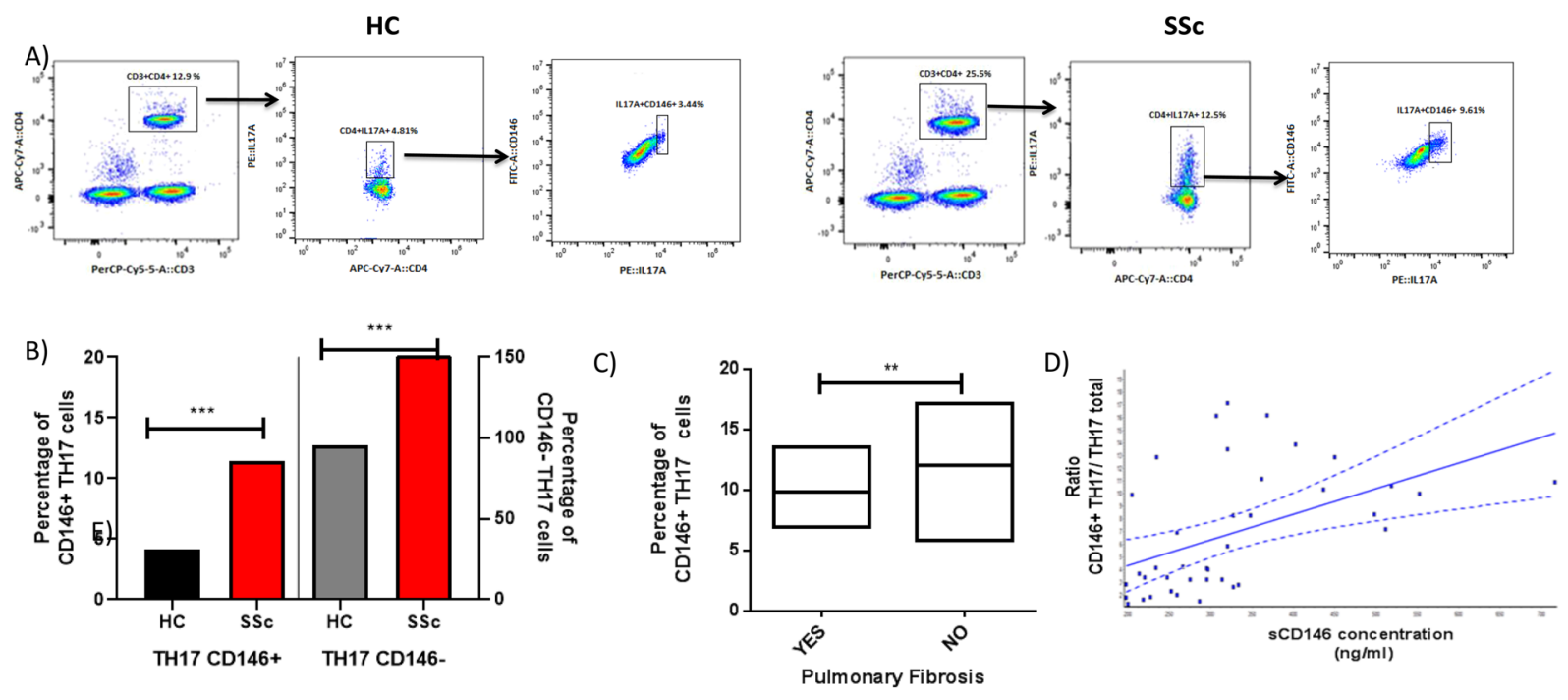

Figure 4. Flow cytometry analysis of TH17 expressing CD146 in Systemic sclerosis. (A) Gating strategy for analysis of CD146 expression on TH17 total cells in HC compared to patients with SSc. (B) The mean percentage of TH17 cells expressing CD146 (CD146+ TH17cells) and non expressing CD146 (CD146- TH17) among HC and patients with SSc: Percentage of TH17 cells expressing CD146 (CD146+ TH17) is higher in case of SSc than in healthy controls $(\mathrm{p}=0.0019)$. (C) The percentage of TH17 cells expressing CD146 (CD146+ TH17) is inversely correlated to pulmonary fibrosis $(\mathrm{p}=0.04)(\mathrm{Yes}=$ have pulmonary fibrosis $/ \mathrm{No}=$ have other involvement than pulmonary fibrosis). (D) A correlation is observed between the ratio (CD146+ TH17/TH17 Total) cells and sCD146 concentration in patients with SSc $(\mathrm{p}=0.0004)$.

significant correlation between SCD146 and IL17A concentrations. This positive correlation could explain the protective role of sCD146, interfering with the counterbalance of inflammation and fibrosis mediated by IL17A.

In patients with autoimmune disease, an increased proportion of TH17 cells were described in the peripheral circulation and at the sites of active inflammation ${ }^{20}$. Several studies have shown that in SSc, TH17 cells could affect the inflammatory response of the disease, and potentially the fibrosis process ${ }^{21}$. Our results confirm 
A)

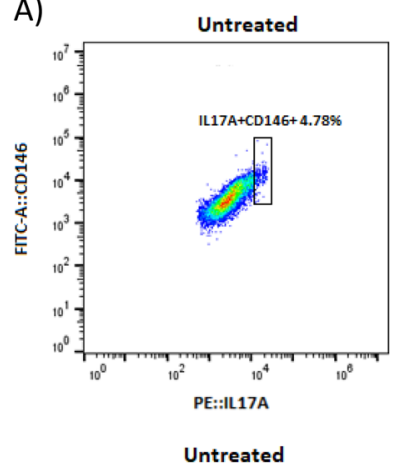

Treated

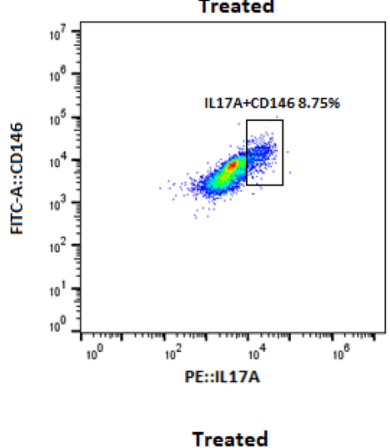

Treated

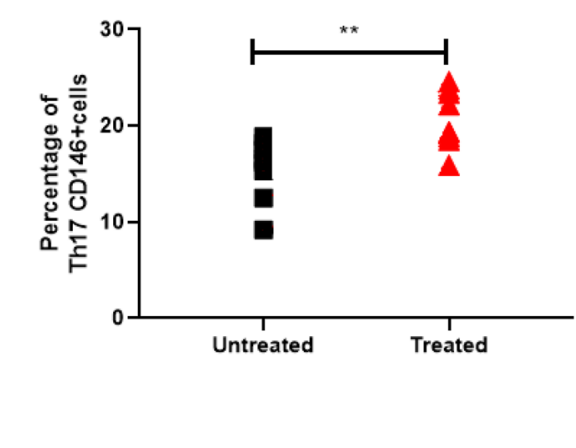

B)

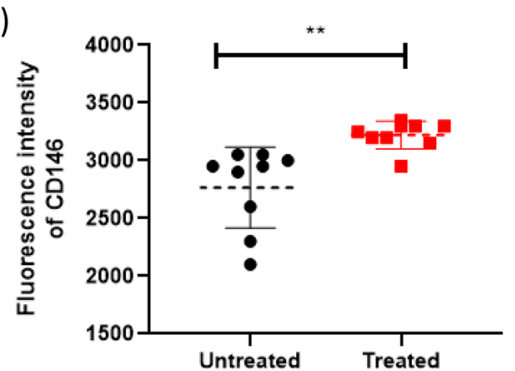

Figure 5. Expression of CD146 on TH17 cells in vitro; Expression of CD146 on TH17 cells was analyzed in peripheral blood mononuclear cells (PBMCs) from healthy controls (HC) and patients with SSc. (A) Flow cytometry analysis of TH17 cells expressing CD146 in PBMCs from patients with SSc treated with sCD146: in each subpopulation, expression of CD146 on TH17 cells was compared between untreated PBMCs (squares) and treated ones (triangles) in patients with SSc. (B) Flow cytometry analysis of TH17 cells expressing CD146 in PBMCs from patients with SSc treated with sCD146: Fluorescence intensity of CD146 is higher on TH17 cells from PBMCs treated with sCD146 compared to untreated ones. (C) The expression of the transcription factor ROR $\gamma$ T mRNA and IL17A mRNA in PBMCs of patients with SSc before and after treatment with sCD146: there is no difference in ROR $\gamma \mathrm{T}$ mRNA expression $(\mathrm{p}=0.7994)$ while IL17A mRNA expression was lower after treatment $(\mathrm{p}=0.0053)$. (D) CD146 mRNA expression in PBMCs of patients with SSc before and after treatment with sCD146: there is an increase in CD146 mRNA expression after treatment $(p=0,0232)$.

a higher percentage of TH17 cells in patients with SSc and show for the first time that this increase is partially due to a higher percentage of TH17 cells expressing CD146. We propose that in patients, the up-regulation of this sub-population could be related to the sera sCD146 increase. In accordance with this hypothesis, we showed an in vitro increase of TH17 cells expressing CD146 after sCD146 treatment. This increase was attributed to an augmentation of CD146 expression since we showed an up-regulation of fluorescence intensity without TH17 proliferation. Such an increase of membrane CD146 expression has been already reported in endothelial cells ${ }^{22}$. We also showed that sCD146 concentrations correlate with the TH17 expressing CD146/TH17 total cells ratio in patients with SSc.

Altogether our results led us to hypothesize that SCD146 could be involved in TH17 cells differentiation and their homing to the site of the disease. Along this line, it has been described that CD146 positive TH17 cells was the sub-population of TH17 cells with an enhanced ability to bind to, and to cross through, vascular endothelium. In addition, CD146 was described as a marker of mouse NK cell maturation ${ }^{23}$. At last, TH17 expressing CD146 are able to migrate from the peripheral circulation to sites of active inflammation ${ }^{24}$. Further studies will be conducted to confirm the role of this sub-population CD146+ TH17 in the fibrotic process, to get more insights into mechanistic pathways and to investigate the potential interest for therapeutic applications.

In conclusion, TH17 cells expressing CD146 could represent a new component of the adaptive immune response and could be potential targets for therapy, opening the way for the generation of new tools for the management of SSc.

Received: 28 June 2019; Accepted: 11 November 2019;

Published online: 27 November 2019

\section{References}

1. van den Hoogen, F. et al. 2013 Classification Criteria for Systemic Sclerosis: An American College of Rheumatology/European League Against Rheumatism Collaborative Initiative: ACR/EULAR Classification Criteria for SSc. Arthritis \& Rheumatism $\mathbf{6 5}$, 2737-2747 (2013).

2. Zhao, W. et al. The status of pulmonary fibrosis in systemic sclerosis is associated with IRF5, STAT4, IRAK1, and CTGF polymorphisms. Rheumatol Int 37, 1303-1310 (2017).

3. Chu, H. et al. Sirtuin1 Protects against Systemic Sclerosis-related Pulmonary Fibrosis by Decreasing Proinflammatory and Profibrotic Processes. Am J Respir Cell Mol Biol 58, 28-39 (2018). 
4. Kaspi, E. et al. Identification of CD146 as a novel molecular actor involved in systemic sclerosis. Journal of Allergy and Clinical Immunology 140, 1448-1451.e6 (2017).

5. Bardin, N., Francès, V., Combes, V., Sampol, J. \& Dignat-George, F. CD146: biosynthesis and production of a soluble form in human cultured endothelial cells. FEBS Letters 421, 12-14 (1998).

6. Bardin, N. et al. S-Endo 1, a pan-endothelial monoclonal antibody recognizing a novel human endothelial antigen. Tissue Antigens 48, 531-539 (1996).

7. Bardin, N. Identification of CD146 as a component of the endothelial junction involved in the control of cell-cell cohesion. Blood 98, 3677-3684 (2001).

8. Bardin, N. et al. CD146 and its Soluble Form Regulate Monocyte Transendothelial Migration. ATVB 29, 746-753 (2009).

9. Kebir, A. et al. CD146 Short Isoform Increases the Proangiogenic Potential of Endothelial Progenitor Cells In Vitro and In Vivo. Circulation Research 107, 66-75 (2010).

10. Bardin, N. et al. Increased expression of CD146, a new marker of the endothelial junction in active inflammatory bowel disease. Inflammatory Bowel Diseases 12, 16-21 (2006).

11. Ito, T. et al. Elevated serum levels of soluble CD146 in patients with systemic sclerosis. Clin Rheumatol 36, 119-124 (2017).

12. Zheng, H., Chen, G., DeLouise, L. A. \& Lou, Z. Detection of the Cancer Marker CD146 Expression in Melanoma Cells with Semiconductor Quantum Dot Label. j biomed nanotechnol 6, 303-311 (2010).

13. Kaspi, E. et al. Identification of soluble CD146 as a regulator of trophoblast migration: potential role in placental vascular development. Angiogenesis 16, 329-342 (2013).

14. Wu, C., Goodall, J. C., Busch, R. \& Gaston, J. S. H. Relationship of CD146 expression to secretion of interleukin (IL)-17, IL-22 and interferon- $\gamma$ by CD $4^{+} \mathrm{T}$ cells in patients with inflammatory arthritis: Cytokine secretion by $\mathrm{CD} 146^{+} \mathrm{CD} 4^{+} \mathrm{T}$ cells. Clin Exp Immunol 179, 378-391 (2015).

15. Castro, G. et al. ROR $\gamma$ t and ROR $\alpha$ signature genes in human TH17 cells. PLoS One 12, e0181868 (2017).

16. Gu, C., Wu, L. \& Li, X. IL-17 family: Cytokines, receptors and signaling. Cytokine 64, 477-485 (2013).

17. Bălănescu, P., Bălănescu, E. \& Bălănescu, A. IL-17 and TH17 cells in systemic sclerosis: a comprehensive review. Romanian Journal of Internal Medicine 55, 198-204 (2017).

18. Amatya, N., Garg, A. V. \& Gaffen, S. L. IL-17 Signaling: The Yin and the Yang. Trends in Immunology 38, 310-322 (2017).

19. Zhou, Y. et al. The elevated expression of TH17-related cytokines and receptors is associated with skin lesion severity in early systemic sclerosis. Human Immunology 76, 22-29 (2015).

20. Bedoya, S. K., Lam, B., Lau, K. \& Larkin, J. TH17 Cells in Immunity and Autoimmunity. Clinical and Developmental Immunology 2013, 1-16 (2013).

21. Truchetet, M.-E., Brembilla, N. C., Montanari, E., Allanore, Y. \& Chizzolini, C. Increased frequency of circulating Th22 in addition to TH17 and Th2 lymphocytes in systemic sclerosis: association with interstitial lung disease. Arthritis Res Ther 13, R166 (2011).

22. Bardin, N. et al. Soluble CD146, a novel endothelial marker, is increased in physiopathological settings linked to endothelial junctional alteration. Thromb Haemost 90, 915-920 (2003)

23. Despoix, N. et al. Mouse CD146/MCAM is a marker of natural killer cell maturation. Eur. J. Immunol. 38, 2855-2864 (2008).

24. Dagur, P. K. et al. MCAM-expressing CD4+ T cells in peripheral blood secrete IL-17A and are significantly elevated in inflammatory autoimmune diseases. Journal of Autoimmunity 37, 319-327 (2011).

\section{Acknowledgements}

We would like to thank: All the members of cytometry platform at the faculty of medicine of Tunis for flow cytometry analysis. All the members of Laboratory of toxins and venoms at the Institute Pasteur Tunis. All the members of internal medicine at the RABTA hospital Tunis, La Timone Hospital Marseille and La conception Hospital Marseille for their cooperation. This work was supported by the Ministry of Higher Education and Scientific Research of Tunisia.

\section{Author contributions}

A.G. wrote the main manuscript text and prepared the figs. N.B., M.R. and M.B.C. checked the manuscript and corrected the figures. X.H., A.S.L. and A.B. helped on the sCD146 manipulations. M.S.K., F.S., M.H.H. and B.G. checked the clinical part of the article. A.D., H.S., A.S., A.A. and B.N. helped on the bench for the manipulations of qPCR and flow cytometry. G.A. helped on the flow cytometry analysis. All the authors reviewed the manuscript.

\section{Competing interests}

The authors declare no competing interests.

\section{Additional information}

Correspondence and requests for materials should be addressed to A.G.

Reprints and permissions information is available at www.nature.com/reprints.

Publisher's note Springer Nature remains neutral with regard to jurisdictional claims in published maps and institutional affiliations.

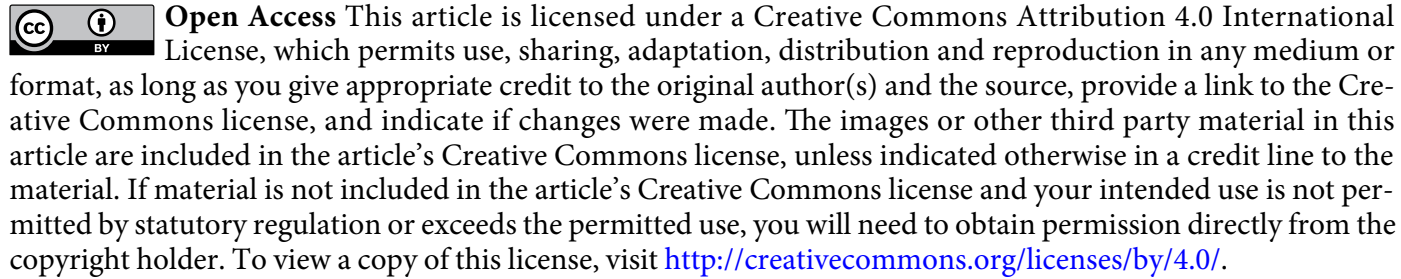

(C) The Author(s) 2019 